\title{
Code of practice for the clinical assessment of licensed medicinal products in general practice
}

\author{
Joint agreement between BMA, RCGP, and ABPI
}

\section{Foreword}

This code of practice offers guidance to the medical profession, pharmaceutical companies, ethical committees, and other interested parties on the clinical evaluation of licensed medicinal products in general practice. It has been agreed jointly by the British Medical Association, the Royal College of General Practitioners, and the Association of the British Pharmaceutical Industry, and the Department of Health and Social Security was kept fully informed of the discussions leading to the development of this document.

The Health Departments are considering with representatives of the medical profession the amendments required to regulations made under the National Health Service Act 1977 and the equivalent provisions applying to Scotland.

The role of ethical committees with regard to clinical evaluation of licensed medicinal products in general practice has been difficult to define because of the substantial variability in their constitution, availability, and function. It was therefore agreed that clinical trials in general practice should not be obstructed by administrative difficulties in obtaining ethical clearance. The British Medical Association has recently suggested a constitution ${ }^{1}$ for ethical committees which, if accepted during discussions with other professional bodies, will ensure better standardisation of the framework and functions of ethical committees. This in turn should mean that approval of a clinical trial protocol by one ethical committee will be recognised and accepted in other areas in the country. Medical practitioners will still be free, however, to seek local ethical committee approval should they so desire. If such local ethical committees have not been constituted or are unable to consider applications for approval within a reasonable time a pharmaceutical company or other sponsoring organisation may seek approval from any ethical committee that has been constituted, and functions, in accordance with the suggestions of the British Medical Association.

Should a clinical trial proposal be rejected by a properly constituted ethical committee, the applicant should be provided in writing with the reasons for the rejection and be given an opportunity to appeal to and appear before that same ethical committee.

It is hoped that all parties concerned will adopt the code of practice as far as is practical immediately after its publication. It should be realised, however, that there may be inevitable delays over the formation of ethical committees after the finalisation of the British Medical Association's proposal on ethical committees for clinical research.

The Department of Health and Social Security is considering with representatives of the medical profession what amendments may be necessary to the National Health Service (General Medical and Pharmaceutical Services) Regulations 1974 in order that a general medical practitioner may accept a fee for work done in respect of a clinical trial conducted in accordance with this code of practice. Also, the Department of Health and Social Security will be negotiating with the Pharmaceutical Services Negotiating Committee on conditions relating to the free supply of medicinal products to patients by the investigators. The interpretation of clauses 5.4 and 10 will be dependent upon the outcome of these discussions.

\section{(1) Introduction}

General practitioner trials that are designed, conducted, and recorded in a scientifically sound manner may contribute to the available information about the safety and efficacy of medicinal products. Clinical trials carried out before a product licence is granted are subject to control or exemption from certification by the licensing authority established under the Medicines Act 1968. The licensing authority takes the advice of the Committee on Safety of Medicines, which is composed of independent experts in medicine, pharmacology, and allied interests. Such clinical trials, therefore, are not the subject of this document.

In the United Kingdom it is recognised that the investigation of medicinal products before licensing should be complemented by further studies after licensing. A pharmaceutical company may have several reasons for inviting general medical practitioners to undertake recorded assessments of licensed medicinal products. The company does not require the approval of the licensing authority to initiate a trial by general practitioners if the uses of the medicinal product are in accordance with the terms of the product licence.

The purpose of this code-the code of practice for the clinical assessment of licensed medicinal products in general practice -is to offer guidance to pharmaceutical companies and members of the medical profession on the procedures to be followed in the organisation of clinical assessments of licensed medicinal products in general practice. The code takes account of the relationship between the general practitioner and the patient and that between the general practitioner and the pharmaceutical company. The code should be read in conjunction with the revised Declaration of Helsinki (Tokyo 1975) on recommendations guiding medical doctors in biomedical research concerning human subjects.

This code has been drawn up by the Association of the British Pharmaceutical Industry in consultation with the British Medical Association, the Royal College of General Practitioners, and the Department of Health and Social Security. The ABPI commends the code to member companies conducting clinical assessments in general practice and requests that they observe the principles contained therein.

\section{CODE OF PRACTICE FOR THE PHARMACEUTICAL INDUSTRY}

Another code-the code of practice for the pharmaceutical industry-governs the promotion of pharmaceutical products to the medical and allied professions. Copies of that code are available on request from the ABPI at 12 Whitehall, London SW1A 2DY. The text of the code is also published in the ABPI Data Sheet Compendium.

Arrangements for a clinical assessment should not constitute or incorporate a method of sales promotion, and if it is considered that the conduct of the assessment may contravene that code of practice the matter may be referred to the code of practice committee for investigation.

(2) Clinical assessment of licensed medicinal products in general practice

There may be a number of substantial reasons for requiring the recorded assessment of the medicinal product outside hospital. These include:

(a) General practice encompasses a wider, more diverse 
population than that allowed by the strict criteria used in controlled trials in hospitals.

(b) The condition of ambulant patients, particularly those at work, may be different from those who are in hospital.

(c) Results of treatment in domiciliary practice may differ considerably from those that would be observed in hospital.

(d) Side effects and idiosyncrasies caused by medication may appear in normal daily situations which would not be observed in hospital.

(e) The general practitioner has knowledge of his patients and their social and physical environments which is not necessarily shared by the hospital physician.

(f) The general practitioner may be in a better position to assess patient compliance and the effects of presentation and packaging of the medicinal product.

(g) The usefulness of some medicinal products in clinical practice requires comparison with existing preparations over prolonged use.

The purpose and objectives of the clinical trials in general practice must be made clear in the protocol.

\section{(3) Company responsibility}

Within the company the responsibility for the organisation of clinical trials in general practice must be that of the medical department alone.

\section{(4) Ethical acceptance}

All clinical trials in general practice to which this code relates must have the protocol approved by an independent and properly constituted ethical committee before the study is begun.

(4.1) Such an ethical committee for clinical research must be constituted according to such guidelines for its establishment as are eventually agreed by the interested parties and which allow for the continuance of those committees which are already in operation and are working well.

(4.2) Other ethical committees for clinical research with similar membership and selection procedures will be acceptable.

(4.3) The role of the ethical committee should be to consider the medical ethics only of the proposed trial and, if applicable, grant approval.

(4.4) The decision of the ethical committee must be made within 35 consecutive days (excluding bank holidays) of the time that the administering staff received the company application.

(4.5) The general practitioner who is invited to participate in the study (the investigator) has the ultimate ethical responsibility for his patients and, if in doubt about the ethics of the study, should resubmit the protocol to an appropriate ethical committee for further consideration.

\section{(5) The protocol}

For each trial the protocol must be approved by a person with suitable medical qualifications who is employed or retained by the supporting company. Where possible, this physician must discuss the protocol with the medical adviser who was concerned with the prelicensing clinical trials.

The following items must be included in the protocol:

(5.1) Introduction-A brief description of the properties of the medicinal product under investigation must be given.

(5.2) Purpose-A precise statement of the objectives of the trial must be included.

(5.3) Design-The choice of clinical trial design must be clearly stated. In trials using more than one treatment any method (including randomisation) for selection of particular patients for a specified treatment must be described.
(5.4) Supply of medication-The trial medicinal product must $\underline{\square}$ always be supplied by the company free of charge, and the 3 arrangements for supplying the investigator must be described. $\stackrel{\mathbb{Q}}{\Omega}$ The method of recording medicinal products supplied to the $c$ investigator and to the patient must be detailed.

(5.5) Therapeutic controls-In controlled studies the method of control and any control product must be identified. The company medical adviser should refer to the notes setting out $\$$ the position under the Medicines Act 1968 (MAL 32-Clinical II Trials Using Marketed Products) when it is proposed to use a control product (see also paragraph 5.3).

(5.6) Patient selection-The total number of patients to be $\overrightarrow{0}$ studied, the number of patients each investigator is to select, $\frac{C}{0}$ the method of selection of these patients, where appropriate the $\overline{\bar{m}}$ criteria for diagnosis of the disease and its severity, and also the $\vec{\nabla}$ patients who are to be excluded must be stated. The number of patients admitted to the trial must be appropriate to provide a $\mathrm{\omega}$ scientifically meaningful result in terms of the defined objectives $\vec{\circ}$ of the study.

(5.7) Consent-The investigator in seeking informed consent $\vec{\omega}$ must be asked to explain to each patient that he or she is $\bar{D}$ being asked to participate in a trial. Where special groups-for example, mentally handicapped, very old people, or children- $-N_{\infty}$ are involved the protocol must state how consent is obtained. The investigator needs to exercise care that the patient is not $\mathscr{G}$ subjected to any form of undue influence to secure this consent. $\omega$ The investigator may wish to refer to the section on obtaining $\vec{N}$ consent of patients described in the BMA Handbook of Medical of Ethics (paragraphs 4.1, 4.4, and 4.5 of the 1981 edition).

(5.8) Dosage-The total daily dose of each treatment and the $\overrightarrow{\vec{J}}$ time and frequency of administration must be stated. Criteria for $\sigma$ changing the dosage must be listed in detail, including clear definititions of satisfactory and unsatisfactory clinical responses.

(5.9) Full prescribing information-A precise statement of the $\vec{\oplus}$ indications, contraindications, precautions, and warnings for any $\omega$ medicinal product being studied must be included.

(5.10) Duration - The duration of the treatment of each patient in the trial and the target duration of the trial as a whole must be defined.

(5.11) Assessments-The method and frequency of assess- $\stackrel{2}{\mathbb{2}}$ ments, the subjective and objective criteria used to establish the $\overrightarrow{\vec{F}}$ efficacy and safety of the medicinal product, and the method of $\frac{1}{3}$ eliciting and recording the findings must be stated.

(5.12) Compliance-Methods of observing and recording that? the medicinal product has been consumed must be described.

(5.13) Adverse reactions - The method of follow-up assessment and investigation of suspected adverse reactions must be described. Investigators must be reminded of the obligation to 3 use their supplies of yellow cards to notify the Committee on: Safety of Medicines (DHSS, Medicines Division, Market 3 Towers, 1 Nine Elms Lane, London SW8 5NQ) of serious or unusual adverse reactions to any medicinal product and of all 5 adverse reactions to a new medicinal product and to inform the $N$ company medical adviser. The company medical adviser has an obligation to inform the Committee on Safety of Medicines of adverse drug reactions and must also, when appropriate, inform $N$ other investigators of any serious or unusual adverse reaction. N

(5.14) Withdrawals - The criteria for withdrawing patientso from the study and the method of recording the reasons for such decisions must be included. The investigator shall not include $\frac{\bar{\Phi}}{\bar{C}}$ or continue to include a patient in a trial unless he is satisfied? that this course is not against the patient's interest. In some trials an independent physician may be required to advise on $\vec{P}$ withdrawals. The procedure used to obtain and record the reasons that caused participation by the patient to be dis-丞 continued must be described. Patients may withdraw themselves from the trial at any time.

(5.15) Record sheets-Adequately designed record sheets for each patient must be provided.

(5.16) Statistical analysis-The method of analysis proposed를 must be outlined. 
(5.17) Trial coordination-The protocol must include the name and address of the company medical adviser responsible for the trial and an out of hours telephone number.

\section{(6) Patient confidentiality}

An individual must not be identifiable from data supplied for statistical or research purposes except when follow up of the patient is a necessary part of the research and the patient has given written consent to disclosure.

\section{(7) Results}

Investigators will be able to see the results appertaining to their patients and to the study as a whole and have the opportunity of commenting before the use of these in part or in whole.

(7.1) Publication-Publication of the results of the trial must be encouraged when possible.

\section{(8) Investigators}

(8.1) The general practitioners whom the company wish to invite to participate in the study must be selected, so far as practicable, so that their practices offer a representative sample consistent with the scientific objectives of the study.

(8.2) The company medical adviser must not invite the participation of general practitioners who do not have the facilities, time, or opportunity to conduct the study according to the protocol or to provide the medical and technical information required.

(8.3) Pressure must not be applied to the investigator to use a medicinal product in any way contrary to his usual prescribing habits.

(8.4) The following must be supplied to each investigator before starting the study:

(a) The protocol.

(b) Record sheets.

(c) The data sheets of the medicinal products concerned.

(d) The code of practice for the clinical assessment of licensed medicinal products in general practice. (e) Declaration of Helsinki.

(f) The name of the ethical committee that has approved the protocol.

\section{(9) Inviting the participation of the general practitioners}

The general practitioner must be approached only by the medical adviser responsible for the protocol or such other person(s) acting on his behalf and whom he is satisfied possess(es) sufficient medical and technical knowledge to present and discuss the study proposed in the protocol. Pressure must not be applied to the general practitioner in seeking his participation in the trial.

\section{(10) Remuneration}

Fees must be realistically related to remuneration for comparable professional work and must be commensurate with the time expended, the complexity of the assessment necessary, and the degree of expertise required of the investigator.

Hospitality should be modest in character and secondary to the main purpose of any meeting.

Fees as well as any other financial arrangements must be agreed in writing before the trial starts and honoured irrespective of the clinical result of the trial, provided that the work is carried out according to the protocol.

\section{(11) Doctor/patient relationship}

(11.1) The company must not seek, directly or indirectly, to make any arrangement that would compromise the overriding commitment of the investigator to his patient.

(11.2) The investigator is free to withdraw a patient from the trial at any time.

\section{Reference}

' British Medical Association. Local ethical committees. Br Med $\mathcal{F} 1981$; 282:1010.
Constrained collective bargaining should be the method for determining pay for National Health Service staff. This is the recommendation of a working seminar organised by the King's Fund. The results of the seminar, which was led by Lady McCarthy and included Health Service chairmen and senior managers as well as experts in pay determination, have been published in $A$ new system for pay determination for the NHS. ${ }^{1}$

The participants believe that unless better ways of determining pay are found quickly disputes in the Health Service such as the ones in 1982 will recur. Five possible options were examined: Diktat (pay awards decided by the government); unfettered, free collective bargaining; automatic indexation; wholesale pay review; and informed, constrained collective bargaining. The first two options were dismissed as unworkable; automatic indexation was considered to be divisive and likely to distort internal reiativities; and wholesale pay review was rejected as unsuitable. That left a system of constrained, collective bargaining, and the report made the following points about this method:

"( $i$ ) It should be based on the principle of comparability.

"(ii) In order to establish the type and method of comparison, a pay board should be set up consisting of five representatives of the staff sides, five representatives of management (including at least one departmental representative), and an independent chairman appointed by the Secretary of State and acceptable to both sides.

"(iii) The board should be assisted by an independent agency which would carry out the actual work producing the comparators. This agency should be the same for the pay board and the pay review body to be set up for nurses and public sector members. "iv) The board should publish annually the results of its work and the range of earnings produced.

"(v) Negotiations should be carried out by the existing functional Whitley Councils, but the bargaining should be constrained by a predetermined range of figures within the published report of the board. We suggest that the interquartile would be the most suitable range.

"(vi) The regional chairmen should together be responsible for formulating the corporate view of NHS management, through consultation with district chairmen and regional groups of officers. These officers should, in turn, be responsible for collecting the views of their district counterparts.

"(vii) The regional chairmen's secretariat should be enlarged by the creation of a pay research unit to collect and analyse crude data so that the regional chairmen can form an overall view of service needs. The pay research unit should also be available to assist the 